\title{
Mastitis diagnostics: Quantitative PCR for Staphylococcus aureus genotype $B$ in bulk tank milk
}

\author{
R. Boss, ${ }^{*}$ J. Naskova, $†$ A. Steiner, ${ }^{*}$ and H. U. Graber ${ }^{\star 1}$ \\ ${ }^{*}$ Clinic for Ruminants, Department of Clinical Veterinary Medicine, Vetsuisse-Faculty, University of Berne, 3001 Switzerland \\ †Agroscope Liebefeld-Posieux Research Station ALP, 3003 Berne, Switzerland
}

\begin{abstract}
A novel real-time quantitative PCR assay for detecting the pathogenic and contagious Staphylococcus aureus genotype B (GTB) in bulk tank milk was developed and evaluated. The detection of this pathogen in bulk tank milk would greatly facilitate its control, as it is responsible for great economic loss in Swiss dairy herds. The assay is based on the simultaneous detection of 3 GTB-typical target sequences, including 2 enterotoxin genes and a polymorphism within the leucotoxin $\mathrm{E}$ gene. A variety of mastitis-associated bacteria was used to validate the assays, resulting in an analytical specificity of $100 \%$ and high repeatability. The analytical sensitivity in milk was $40 \mathrm{cfu} / \mathrm{mL}$. An exponential association between simulated cow prevalence and quantitative PCR result was observed. An initial field study revealed 1 GTB-positive herd among the 33 studied herds. This novel assay for bulk tank milk analysis is suitable for routine purposes and is expected to be an effective tool for minimizing Staph. aureus GTB in Swiss dairy herds.
\end{abstract}

Key words: bulk tank milk, mastitis, polymerase chain reaction, Staphylococcus aureus

\section{INTRODUCTION}

Staphylococcus aureus is the most common cause of contagious mastitis in cattle worldwide (Schällibaum, 1999; Zecconi et al., 2005). In Switzerland, it causes great economic loss (Kirchhofer et al., 2007); therefore, control of this pathogen would be beneficial. Unfortunately, conventional, routine bacteriology as a diagnostic tool is not entirely satisfactory, because the overall diagnostic sensitivity in single milk samples reaches just $79.9 \%$ (21.4 to 100\%; Studer et al., 2008). Consequently, 3 consecutive samples are necessary to achieve a satisfactory diagnostic sensitivity (Sears et al., 1990), but it is often too expensive, so routine testing normally

Received March 11, 2010.

Accepted September 12, 2010.

${ }^{1}$ Corresponding author: hans.graber@knp.unibe.ch is accomplished with a single analysis. From a clinical point of view, this approach is not acceptable, as many cases of mastitis remain undetected, making the control of Staph. aureus mastitis difficult.

Graber et al. (2007) developed a highly sensitive and specific assay to detect Staph. aureus in raw milk samples. Its potential for automation in routine examinations is the basis for a wide range of use. This assay is >500 times more sensitive than conventional bacteriology and is highly specific for Staph. aureus (100\%). It was evaluated in a longitudinal field study by Studer et al. (2008) and showed a diagnostic sensitivity of $99.4 \%$ and a diagnostic specificity of $97.1 \%$.

In studies by Fournier et al. (2008) and Graber et al. (2009), various genotypes of Staph. aureus with different virulence and pathogenicity factors were identified and described. Genotype B (GTB) and genotype C (GTC) were predominant in Swiss dairy herds, whereas the remaining genotypes (GTOG) were rarely found. Genotype B was related to high contagiousness and increased pathogenicity, causing herd problems with cow prevalence up to $87 \%$ (Graber et al., 2009). By contrast, GTC and GTOG were found in infections of single cows.

Genotyping by Fournier et al. (2008) showed a high association between genotypes and virulence gene patterns. Among others, GTB was characterized by the presence of the Staph. aureus enterotoxin genes A (sea) and $\mathrm{D}(\mathrm{sed})$, and by a polymorphism within the leucotoxin $\mathrm{E}$ gene (lukE), caused by a point mutation, and called lukEB. Fournier et al. (2008) correctly identified $77 \%$ of GTB isolates by sea, $87 \%$ by sed, and $100 \%$ by lukEB. Correct negative results for non-GTB strains were observed in $100 \%$ of isolates for sea, $94 \%$ for sed, and $87 \%$ for lukEB.

As shown by Fournier et al. (2008) and Graber et al. (2009), Staph. aureus GTB infects many cows in a herd and requires infected herds to be sanitized to reduce SCC at herd level. From clinical experience, we know that proper sanitation alone takes $1 \mathrm{yr}$ and is expensive because of treatment costs, loss of milk, culling, replacements, additional work, and veterinary support. To minimize IMI caused by Staph. aureus GTB at the herd 
and country levels, analysis of bulk tank milk (BTM) to detect and monitor GTB-positive herds would be the most efficient and economical method. Screening of BTM is a convenient, fast, and comparatively cheap method.

To improve the diagnostics for Staph. aureus as a mastitis pathogen, the goal of this study was to develop a novel assay to detect GTB in BTM. With the triple detection of lukEB, sea, and sed by real-time quantitative PCR (QPCR), this new method showed a high analytical sensitivity and specificity as well as a high repeatability.

\section{MATERIALS AND METHODS}

The development and validation of the assay specific for Staph. aureus GTB were undertaken according to guidelines proposed by the World Organization for Animal Health (OIE, 2008), adapted for QPCR. In particular, the analytical sensitivity and specificity of the assay as well as its repeatability were evaluated using a variety of bacterial DNA samples. The assay was then applied on simulated and real BTM samples.

\section{Sequencing of the sea and sed Target Genes}

In addition to lukEB, the genes sea and sed were chosen as QPCR targets because they have been shown to be typical for Staph. aureus GTB (Fournier et al., 2008; Graber et al., 2009). The DNA sequencing was performed to look for conserved regions of the sea and sed target genes. For both genes, 9 epidemiologically independent bovine strains of Staph. aureus were selected from our strain collection, and DNA was extracted from single colonies as described below. The unpurified lysates were diluted 1:100 in $\mathrm{H}_{2} \mathrm{O}$ and were subjected to standard PCR for sea and sed (see below), respectively. The resulting amplicons were purified using the QIAquick PCR purification kit (Qiagen AG, Hombrechtikon, Switzerland).

The DNA concentration of the eluates was measured by spectroscopy using a Nanodrop ND-1000 spectrometer (Nanodrop Technologies, Rockland, DE); 100 ng was then added to a total volume of $10 \mu \mathrm{L}$ containing 20 pmol of the sea or sed gene sequencing primers, respectively (Table 1). The sequencing procedures were done by the sequencing facility of Microsynth AG (Balgach, Switzerland).

\section{Bacterial Strains}

The DNA samples of udder pathogens with known identity were tested. Strains of Staph. aureus were obtained from former studies (Graber et al., 2007;
Fournier et al., 2008) and initially isolated from bovine milk with spontaneous IMI. In total, there were 33 strains of Staph. aureus GTB, 18 strains of Staph. aureus GTC, and 20 strains of Staph. aureus GTOG, with the genotypes A, F, F', G, I, J, K, L, M (Fournier et al., 2008), and newly detected genotypes $\mathrm{J}^{\prime}, \mathrm{S}, \mathrm{S}^{\prime}$, and $\mathrm{T}$ resulting from the present study (see below). For CNS, 26 IMI-associated isolates were reused from the study of Graber et al. (2007) and 9 strains with known identity were obtained (Deutsche Sammlung von Mikroorganismen und Zellkulturen GmbH, Braunschweig, Germany): Staph. hemolyticus (DSM 20263), Staph. warneri (DSM 20316), Staph. simulans (DSM 20322), Staph. intermedius (DSM 20373), Staph. chromogenes (DSM 20454), Staph. hyicus (DSM 20459), Staph. caprae (DSM 20608), Staph. xylosus (DSM 20266), and Staph. sciuri ssp. sciuri (DSM 20345). Two strains each of the following pathogens were additionally included: Streptococcus agalactiae, Strep. uberis, Strep. dysgalactiae ssp. dysgalactiae, and Enterococcus faecalis, all identified by API 20 Strep (Biomérieux Suisse s.a., Geneva, Switzerland), 1 isolate of Escherichia coli identified by API $20 \mathrm{E}$, and 1 E. coli ATCC 8739 (American Type Culture Collection, Manassas, VA).

The bacteria were grown on 5\% sheep blood agar plates (BA; Biomérieux Suisse s.a.) or brain heart agar plate (BH; Merck, Berne, Switzerland) for Strep. uberis and Strep. dysgalactiae. The plates were incubated at $37^{\circ} \mathrm{C}$ for $24 \mathrm{~h}$.

\section{DNA Extraction from Agar Plates and Purification}

For extraction of bacterial nucleic acids (NA) containing both DNA and RNA, single colonies were picked from the $\mathrm{BA}$ or $\mathrm{BH}$ agar plates and inoculated in 100 $\mu \mathrm{L}$ of $10 \mathrm{~m} M$ Tris/ $\mathrm{HCl}, 10 \mathrm{~m} M$ EDTA $(\mathrm{pH}=8.5)$. The samples were incubated at $95^{\circ} \mathrm{C}$ for $10 \mathrm{~min}$ for lysis and immediately placed on ice.

To obtain purified DNA, $30 \mu \mathrm{L}$ of the lysate was added to a mixture containing $150 \mu \mathrm{L}$ of $25 \mathrm{~m} M$ Tris/ $\mathrm{HCl}, 10 \mathrm{~m} M$ EDTA $(\mathrm{pH}=7.2), 1.2 \times 10^{5} \mathrm{U}$ of lysozyme (Merck), $8 \mu \mathrm{L}$ of mutanolysine $(2,000 \mathrm{U} / \mathrm{mL}$; Sigma, Buchs, Switzerland), and $2 \mu \mathrm{L}$ of DNase-free RNase (Roche Diagnostics AG, Rotkreuz, Switzerland). After incubation at $37^{\circ} \mathrm{C}$ for $30 \mathrm{~min}, 200 \mu \mathrm{L}$ of binding buffer and $40 \mu \mathrm{L}$ of proteinase $\mathrm{K}$ of the high pure PCR template preparation kit (Roche) were added and the samples processed and eluted in $100 \mu \mathrm{L}$ of elution buffer as described by the manufacturer.

DNA concentrations were measured by spectroscopy (Nanodrop). The $260 \mathrm{~nm}: 280 \mathrm{~nm}$ ratio had to be $\geq 1.80$, otherwise the DNA extraction was repeated. Samples were diluted to $5 \times 10^{3}$, and 100 molecules $/ 3.5 \mu \mathrm{L}$ for Staph. aureus GTB samples or $5 \times 10^{5}, 1.11 \times 10^{4}$ 
GelRed stock reagent (Biotium Inc., Hayward, CA) to $60 \mathrm{~mL}$ of hot gel solution. Bands were visualized using a standard UV transilluminator $(312 \mathrm{~nm})$.

\section{Genotyping of Staph. aureus}

Genotyping of nuc-positive isolates was performed as described by Fournier et al. (2008). In particular, the $16 \mathrm{~S}$ to $23 \mathrm{~S}$ rRNA intergenic spacer region was amplified by PCR (RS-PCR) using the primers of Jensen et al. (1993). The amplicons were then visualized with the miniaturized electrophoresis system, DNA 7500 Chip (Agilent Technologies, Basel, Switzerland). Newly found genotypes were named continuously according to Fournier et al. (2008).

\section{Real-Time Quantitative PCR}

Primers and probes for QPCR were designed with OLIGO 6 software (Molecular Biology Insights Inc., Cascade, CO) and are listed in Table 1. The probes for sea and sed were MGB labeled with 6-carboxyfluorescein at the $5^{\prime}$ end and a confidential dark quencher at the $3^{\prime}$ end synthesized by Applied Biosystems (Rotkreuz, Switzerland). All other primers and probes were synthesized by Microsynth. The probe for lukEB contained 6 locked nucleic acids at the positions indicated in Table 1. It was labeled with 6-carboxyfluorescein at the $5^{\prime}$ end and with BHQ-1 at the $3^{\prime}$ end. The sense primer QlukEB-S specifically detects the point mutation at nucleotide 132 of the lukE gene, typical for Staph. aureus GTB (Fournier et al., 2008; Graber et al., 2009). The primers and probes for nuc- and canine distemper virus gene N (CDV-N)-QPCR were the same as described by Graber et al. (2007).

The QPCR reactions were run in a total volume of $25 \mu \mathrm{L}$ containing $1 \times$ Roche LightCycler 480 Probes Master (Roche). Considering QPCR for nuc, sea, and sed, the concentrations for both primers and the probe were 600 and $100 \mathrm{n} M$, respectively. For lukEB-QPCR, the concentrations were 300 and $200 \mathrm{n} M$, respectively, and for CDV-N-QPCR, the concentrations were 900 and $300 \mathrm{n} M$, respectively. In the case of bacteriological DNA samples obtained from agar plates and enterotoxin gene amplicons prepared by standard PCR, the QPCR reaction mix was additionally spiked with NA of $1.5 \times 10^{9}$ cfu of Lactobacillus casei to simulate extraction from milk. Finally, $3.5 \mu \mathrm{L}$ of template (NA, purified DNA, enterotoxin gene amplicons) was added to the QPCR mix.

The QPCR cycling started with an initial step of $95^{\circ} \mathrm{C}$ for $10 \mathrm{~min}$, followed by 45 cycles of $95^{\circ} \mathrm{C}$ for $15 \mathrm{~s}$, and $60^{\circ} \mathrm{C}$ for $1 \mathrm{~min}$. The QPCR was carried out in a Rotor-Gene 3000 real-time thermal cycler (Corbett Life Science, Mortlake, Australia).
The QPCR for all genes was run in duplicate. Results were considered positive if both reactions were positive. If only 1 reaction showed a positive result or the difference of the 2 cycle-to-threshold values was more than 2 cycles, the QPCR was repeated. A reaction was considered negative for all of the targets analyzed if amplification resulted in a value $<10$ copies/reaction. For each assay and each gene, a standard was included (in duplicates) ranging between 10 to $10^{5}$ copies/assay. In the case of the nuc gene, 1 molecule was considered as 1 staphylococcal cell equivalent (Graber et al., 2007).

\section{Assay Controls}

Various controls were included to monitor the whole procedure. To evaluate the preparation of Staph. aureus from milk, 1 sample of sterile raw milk negative for lukEB, sea, sed, and nuc, as well as 1 raw milk sample spiked with $10^{5} \mathrm{cfu} / \mathrm{mL}$ of Staph. aureus GTB positive for the 3 target sequences and $n u c$, were always coprocessed with the test samples. If the raw milk sample showed amplification or if the spiked sample was not within the adequate range ( \pm twice the expected nontransformed value), all test samples of the affected series were prepared again.

For each QPCR run, no-template controls were included. If the results were positive, the run was repeated.

To check for QPCR inhibitors present in NA, the samples were analyzed by an additional QPCR containing CDV-N gene amplicons as an internal control, which is detected by CDV-N-specific primers and a fluorescent probe (Graber et al., 2007). Negative QPCR results for the target sequences required a positive CDV-N QPCR result to exclude inhibitors.

\section{Composite Cow Milk Samples}

The 2,473 quarter milk samples of 629 dairy cows were reused from other studies (Moret-Stalder et al., 2009; Graber et al., 2009). The samples originated from 36 farms in the western half of Switzerland. Herds characterized by a quarter prevalence of Staph. aureus $\geq 10 \%(\mathrm{n}=11)$ comprised 282 cows, whereof 127 were positive for GTB (Graber et al., 2009). Herds without Staph. aureus GTB-secreting cows $(\mathrm{n}=25)$ comprised 347 cows. In these herds, the quarter prevalence of Staph. aureus was always $<10 \%$ (Graber et al., 2009). The quarter milk samples had been collected aseptically using standard procedures (National Mastitis Council, 1999), transported on ice, and stored at $-20^{\circ} \mathrm{C}$. Breed and herd size were not considered.

For each cow, the corresponding quarter milk samples were pooled to create a composite cow milk (CCM) 
sample by adding equal volumes of prewarmed $\left(37^{\circ} \mathrm{C}\right)$ milk to a common tube.

From former studies, bacteriological results, SCC, and Staph. aureus genotypes obtained by RS-PCR (Fournier et al., 2008; Graber et al., 2009) were available.

\section{Bulk Tank Milk Samples}

An overview over all BTM samples is given in Table 2. The GTB-positive CCM samples $(\mathrm{n}=127)$ were used to create (1) simulated BTM with a GTB cow prevalence of $5 \%(\mathbf{p 5 B T M} ; \mathrm{n}=24)$ and $(2)$ simulated BTM with a GTB cow prevalence ranging from 18 to 95\% (pxBTM; $\mathrm{n}=40$ ) based on the findings of Graber et al. (2009) in real herds. By assembling constant volumes of randomly attributed GTB-positive CCM samples (sampling procedure with putting back) and by adding a calculated volume of GTB-free milk, the attributed cow prevalence was achieved. To create simulated control herds (cBTM; $\mathrm{n}=25)$, equal volumes of GTB-negative CCM were combined. For assembling, all milk samples were prewarmed to $37^{\circ} \mathrm{C}$.

Real BTM (rBTM) was collected from 33 herds (12 to 58 cows per herd) in the area of Berne, Switzerland, all of which were attended by the same practicing veterinarian. Without any knowledge about cow udder health status, the first 33 farmers who agreed were visited. After stirring the milk tank for $10 \mathrm{~min}$, BTM was collected according to NMC (1999) guidelines, transported on ice, and stored at $-20^{\circ} \mathrm{C}$ until further use.

Preparation of bacteria from milk using $L$. casei and NA extraction were performed according to Graber et al. (2007) with the following modification: for cell lysis, the pelleted bacteria were resuspended in $150 \mu \mathrm{L}$ of 25 $\mathrm{m} M$ Tris $/ \mathrm{HCl}, 10 \mathrm{~m} M$ EDTA $(\mathrm{pH}=7.2)$, incubated at $95^{\circ} \mathrm{C}$ for $10 \mathrm{~min}$, and immediately placed on ice. The final elution volume was $200 \mu \mathrm{L}$.

\section{Bacteriology}

Conventional bacteriology from rBTM samples was performed as proposed by the NMC (1999). In particular, the following agar plates were used: BA for a general overview and total bacterial growth, modified Edwards agar (ED; Oxoid, Basel, Switzerland) for the detection of streptococci and related organisms, Vogel-Johnson agar (VJ; Becton Dickinson AG, Basel, Switzerland) for the identification of staphylococci, and MacConkey agar (MC; Oxoid) for the detection of Enterobacteriaceae, including coliforms. The prewarmed $\left(37^{\circ} \mathrm{C}\right)$ rBTM samples were intensively vortexed, and $10 \mu \mathrm{L}$ of each sample was then spread over each of the different agar plates using sterile triangle spatulas. After incubation for $24 \mathrm{~h}$ and $48 \mathrm{~h}$, respectively, colonies were
Table 2. List of all bulk tank milk (BTM) samples as an overview

\begin{tabular}{lccc}
\hline $\begin{array}{l}\text { BTM } \\
\text { sample }^{1}\end{array}$ & $\mathrm{n}$ & $\begin{array}{c}\text { Cow prevalence } \\
\text { of GTB }\end{array}$ & $\begin{array}{c}\text { Herd size } \\
\text { (cows) }\end{array}$ \\
\hline cBTM & 25 & 0 & $4-44$ \\
p5BTM & 24 & 5 & 20 \\
pxBTM & 40 & $18-95$ & $11-60$ \\
rBTM & 33 & Unknown & $12-58$ \\
\hline
\end{tabular}

${ }^{1} \mathrm{cBTM}=$ control herds (simulated); p5BTM $=$ simulated problem herds with $5 \%$ cow prevalence; pxBTM = simulated problem herds with different cow prevalences; rBTM $=$ real BTM.

${ }^{2} \mathrm{GTB}=$ Staphylococcus aureus genotype B.

counted as proposed by the NMC (1999). For the final results, growth after $48 \mathrm{~h}$ was considered and noted as semiquantitative values: -, no growth, 0 colonies; +, weak growth, 1 to 10 colonies; ++ , medium growth, 10 to 100 colonies; +++ , intense growth, $>100$ colonies.

Additionally, Chromagar Staph. aureus plates (CHROMagar, Paris, France) specific for Staph. aureus were inoculated with $100 \mu \mathrm{L}$ of 11 selected $\mathrm{rBTM}$ samples to search for other genotypes. After incubation at $37^{\circ} \mathrm{C}$ for $24 \mathrm{~h}, 10$ mauve colonies per plate were subcultured on BA. One colony of each BA plate was then picked and checked for Staph. aureus using nuc gene PCR (see above), and all positives were genotyped by RS-PCR as described above.

\section{Statistical Analysis}

Data are expressed as absolute numbers, percentages, or as log-transformed values (logarithm to the basis 10). Robust nonlinear regression analysis was performed, with the QPCR results $\left[\log _{10}\right.$ (copies/reaction)] forming the independent variable, and the simulated cow prevalence of a herd (\%) acting as the dependent variable. The model was selected that produced the maximal coefficient of determination $\left(\mathrm{R}^{2}\right)$, resulting in an exponential dependency between both variables. For all of the statistical analyses, the Systat 12 software package (Systat Software Inc., Richmond, CA) was used. Values of $P<0.05$ were considered significant.

\section{RESULTS}

\section{Target Gene Analysis}

To obtain a solid basis for selecting primers that allow for consistent sea and sed gene amplifications of bovine strains, 9 epidemiologically unrelated Staph. aureus isolates obtained from bovine mastitic milk were sequenced for both genes.

For the sea gene, all 9 strains showed a 100\% identity within the 448 sequenced nucleotides. In addition, they were $100 \%$ identical to the reference sea sequence (GenBank BX571857). 
Table 3. Examined DNA samples and their real-time quantitative PCR (QPCR) results for the 3 targets and the resulting analytical specificities

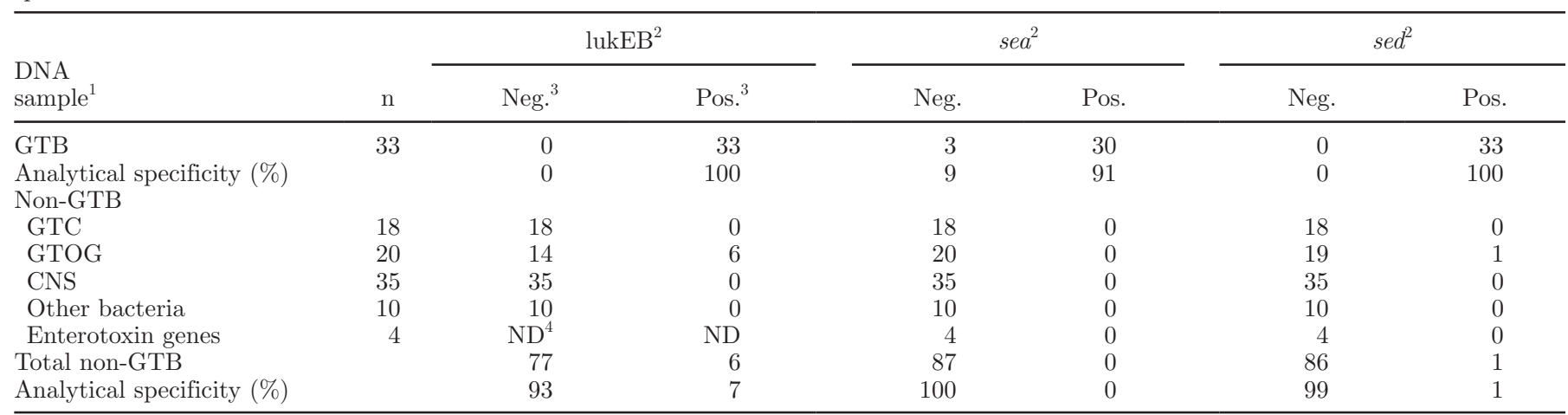

${ }^{1} \mathrm{GTB}=$ Staphylococcus aureus genotype B; GTC $=$ Staph. aureus genotype C; GTOG = other genotypes than GTB and GTC

${ }^{2}$ Target genes: lukEB = point mutation within leucotoxin E gene, typical of GTB; sea = Staph. aureus enterotoxin gene A; sed = Staph. aureus enterotoxin gene D.

${ }^{3} \mathrm{Neg} .=$ number of negative QPCR results; Pos. = number of positive QPCR results.

${ }^{4} \mathrm{ND}=$ not determined.

Considering the sed gene, comparison of the 9 bovine sequences (each 560 nucleotides long) to the reference sed sequence (GenBank M28521) resulted in similarities ranging from 98.4 to $99.6 \%$. All 9 strains showed a transition at nucleotide 674 , resulting in a substitution of Arg to Lys and a transversion at nucleotide 631, leading to an exchange of Pro against Ala. Two of the 9 strains showed further transitions at nucleotides 499 (Asp to Asn), 674 (Arg to Lys), 932 (Ser to Phe), 998 (Arg to Gln), and 1005 (silent mutation), and transversions at nucleotides 631 (Pro to Ala), 667 (Ile to Lys), 812 (Asn to Ile), and 860 (Lys to Thr). Two other strains further showed a deletion at nucleotide 805 , resulting in a chain termination 6 AA downstream.

Based on these findings, 2 primers and a fluorescent probe for QPCR were designed for both genes (Table 1 ). They were expected to detect the sea and sed genes of all the bovine and human Staph. aureus isolates specifically.

Specific amplification also was expected for the GTB-typical polymorphism of lukE (point mutation). As based on previous studies using restriction-length polymorphism and DNA sequencing for differentiation, the point mutation could be detected by the lukEBQPCR sense primer designed for this study (Table 1).

\section{Real-Time Quantitative Assay Performance}

Using serial dilutions of purified lukEB, sea and sed amplicons, the assays were shown to give log-linear results between 10 and $10^{5}$ copies/assay. For this range, the coefficient of correlation of each of the 3 QPCR was 0.999. The amplification efficiency was 1.02 for lukEB and sed, and 1.01 for sea and nuc, as measured on the gradient.

\section{Analytical Specificity of the Single-Target QPCR}

For lukEB-QPCR, the analytical specificity was $93 \%$ (77/83; Table 3). Six out of 20 Staph. aureus GTOG strains tested positive for lukEB. All of the Staph. aureus GTC, CNS, and other bacteria were lukEB-QPCR negative. In addition, all of the Staph. aureus GTB strains tested positive for lukEB.

For sea-QPCR, the analytical specificity was $100 \%$ (87/87; Table 3). All of the Staph. aureus GTC, GTOG, CNS, and other bacteria, and all of the other enterotoxin genes were sea-QPCR negative. In addition, 30 out of 33 Staph. aureus GTB strains tested positive for sea $(91 \%)$.

For sed-QPCR, the analytical specificity was $99 \%$ (86/87; Table 3). One out of 20 Staph. aureus GTOG strains tested positive for sed. All of the Staph. aureus GTC, CNS, and other bacteria, and all of the other enterotoxin genes were sed-QPCR negative. In addition, all of the Staph. aureus GTB strains tested positive for sed.

\section{Analytical Specificity of the Assay}

Using the analytical specificity of the single targets (Table 3), the probabilities of observing different combinations of QPCR results were calculated (Table 4) given that a milk sample was truly Staph. aureus GTB positive or negative, respectively. For a sample positive for the 3 targets (lukEB + , sea + , sed + ), the probability of being GTB positive was $0.909(1 \times 0.909 \times$ $1)$; the probability of being a non-GTB was $0(0.072 \times$ $0 \times 0.012)$. For lukEB + , sea-, and sed + samples, the probablities were 0.091 and 0.001 , respectively. All of the other combinations were typical of GTB-negative samples or were improbable (Table 4). 
Table 4. Discrimination: probabilities of milk samples containing Staphylococcus aureus genotype B (GTB) or non-GTB, combining the real-time quantitative PCR (QPCR) results for the 3 targets

\begin{tabular}{|c|c|c|c|c|}
\hline \multicolumn{3}{|c|}{ Combination of QPCR results ${ }^{1}$} & \multicolumn{2}{|c|}{ Probabilities } \\
\hline lukEB & sea & sed & GTB & Non-GTB \\
\hline+ & + & + & 0.909 & 0 \\
\hline+ & - & + & 0.091 & 0.001 \\
\hline+ & + & - & 0 & 0 \\
\hline+ & - & - & 0 & 0.071 \\
\hline- & + & + & 0 & 0 \\
\hline- & - & + & 0 & 0.011 \\
\hline- & + & - & 0 & 0 \\
\hline - & - & - & 0 & 0.918 \\
\hline
\end{tabular}

${ }^{1}$ lukEB = point mutation within leucotoxin E gene, typical of GTB; sea = Staph. aureus enterotoxin gene A; sed $=$ Staph . aureus enterotoxin gene $\mathrm{D} ;+$ (positive) $=$ amplification over limit of detection; $-($ negative $)=$ no amplification.

\section{Analytical Sensitivity}

The analytical sensitivity of the different QPCR assays was evaluated by serial 1:10 dilutions of milk spiked initially with $10^{5} \mathrm{cfu} / \mathrm{mL}$ of Staph. aureus GTB positive for the 3 target sequences. The limits of detection were $40 \mathrm{cfu} / \mathrm{mL}$ milk for lukEB, $100 \mathrm{cfu} / \mathrm{mL}$ for sea, and $10 \mathrm{cfu} / \mathrm{mL}$ for sed.

\section{Repeatability}

The intraassay variability was determined by simultaneous extraction $(\mathrm{n}=8)$ of the same milk sample spiked with $10^{5} \mathrm{cfu} / \mathrm{mL}$ of Staph. aureus GTB. The samples were completely analyzed (bacteria preparation, NA extraction, QPCR) 8 times for the targets (i.e., lukEB, sea, and sed). The coefficient of variation was $2.9 \%$ for lukEB, $0.9 \%$ for sea, and $1.6 \%$ for sed ( $\log _{10}$-transformed values). The interassay variability was evaluated by a complete analysis of milk samples $(\mathrm{n}=10)$ spiked with $10^{5} \mathrm{cfu} / \mathrm{mL}$ of Staph. aureus GTB on 10 different days. The coefficient of variation was $1.7 \%$ for lukEB, $2.1 \%$ for sea, and $1.8 \%$ for sed $\left(\log _{10^{-}}\right.$ transformed values).

\section{Relationship Between Gene Copy Numbers and Colony Forming Units}

To compare the number of the copies of sea, sed, and lukEB genes to the copy number of the nuc gene, which is a true estimation of the number of Staph. aureus cells, a GTB strain was cultured in tryptic soy broth (Becton Dickinson) at $37^{\circ} \mathrm{C}$ overnight. The DNA extraction and QPCR for the corresponding genes were performed as described above. Compared with the nuc gene, an approximate 4-fold and an approximate 3- to 4 -fold increase was observed for lukEB and sed, respectively. In case of sea, the ratio was approximately 1 .

\section{Bulk Tank Milk Samples}

Overall, an exponential dependency $\left(\mathrm{R}^{2}=0.861 ; P<\right.$ 0.001) was observed between GTB cow prevalence of a herd and the logarithmic lukEB-QPCR value (number of copies per reaction) measured in simulated BTM samples (Figure 1). All cBTM samples $(\mathrm{n}=25)$ were tested negative for lukEB and appear as a single point in the intersection of the axes (Figure 1). All p5BTM samples $(\mathrm{n}=23)$ were tested positive for lukEB and were located within a wide range varying from 69 to $7.67 \times 10^{3}$ copies/reaction. One value was an extreme outlier $\left(6.6 \times 10^{4}\right.$ copies/reaction $)$ and was therefore not considered for further analyses. All pxBTM samples (n $=40$ ) tested positive for lukEB and ranged from 2.61 $\times 10^{3}$ to $7.93 \times 10^{5}$ copies/reaction.

Thirty-three rBTM samples were examined by QPCR for the 3 targets, as well as for nuc (Table 5). One rBTM sample (3\%) was identified as positive for Staph. aureus GTB because of its positive QPCR results for all targets (i.e., lukEB, sea, and sed). Twenty rBTM samples $(61 \%)$ were negative for all targets. The 12 remaining rBTM samples $(36 \%)$ were positive for 1 or 2 targets.

All rBTM samples positive for lukEB- and nucQPCR ( $\mathrm{n}=11)$ were furthermore reverse examined; for each sample, 10 mauve single colonies were selected from Chromagar and subcultured on BA followed by genotyping all those colonies that were found to be nuc positive by standard PCR $(\mathrm{n}=72)$. In total, 8 different genotypes were found (i.e., A, B, C, and F, and the newly found genotypes $\mathrm{J}^{\prime}, \mathrm{S}, \mathrm{S}^{\prime}$, and T; Table 5). Considering farm No. 17, all isolates were confirmed as GTB by genotyping. In 2 rBTM samples, more than 1 genotype was found, and in other 2 samples, none of the colonies was positive for nuc (Table 5).

Bacteriological examination using $10 \mu \mathrm{L}$ of milk revealed mid-range to intense growth on $\mathrm{BA}$ for all 33 


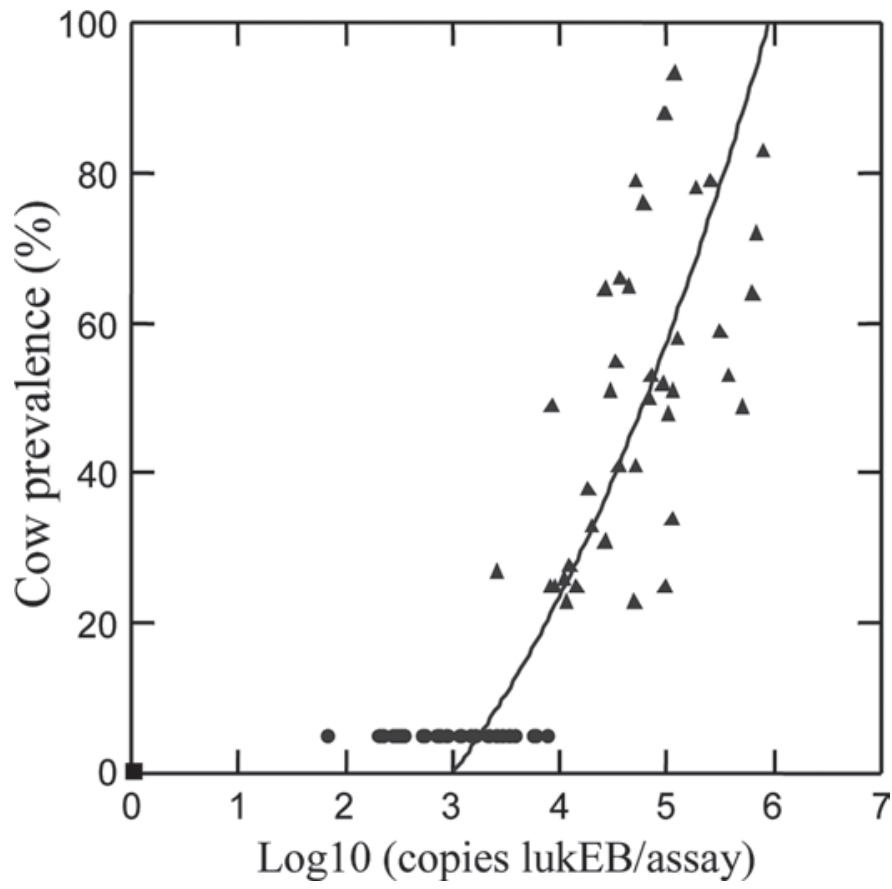

Figure 1. Staphylococcus aureus genotype B (GTB): correlation between cow prevalence in simulated bulk tank milk samples and the corresponding real-time quantitative PCR results ( $\log _{10}$-transformed) for lukEB. Robust nonlinear analysis resulted in $\mathrm{R}^{2}=0.861(P<$ 0.001). lukEB $=$ point mutation within leucotoxin E gene, typical of GTB; control herds (cBTM; $\mathrm{n}=25$; no GTB secreting cows); problem herds (p5BTM; $\mathrm{n}=23$; cow prevalence of $5 \%$ ); $\boldsymbol{\Delta}$ problem herds (pxBTM; $\mathrm{n}=40$; percentage of GTB secreting cows ranging from 18 to $95 \%)$.

rBTM samples. In the majority of the samples, Streptococcus spp. (79\%) and Staphylococcus spp. (88\%) were observed. Enterobacteriaceae were found in $18 \%$ of the samples. Only 8/29 (28\%) samples with growth on VJ were positive for nuc QPCR, but all nuc-positive samples showed staphylococcal growth on VJ agar.

The BTM of the GTB-positive farm (No. 17) showed intense growth on BA, medium growth on VJ, weak growth on ED, and no growth on MC agar.

\section{DISCUSSION}

The present study describes a novel QPCR-based assay to detect Staph. aureus GTB in BTM. The assay is highly sensitive and specific for this contagious mastitis pathogen and is characterized by a small intra- and interassay variability. It includes preparation of bacteria from milk as described by Graber et al. (2007) and QPCR for the 3 GTB-typical targets lukEB, sea, and sed (Fournier et al., 2008; Graber et al., 2009). A BTM sample is GTB-positive if QPCR for lukEB and sed and/or sea generates a positive result each $\geq 10$ copies/reaction. Quantification is based on the lukEB target. The BTM was used as it is the most efficient and economical way of detecting and monitoring GTBpositive herds.

So far, BTM analyses for Staph. aureus using conventional bacteriological examination were of limited value as only a weak association between cfu of Staph. aureus measured in BTM and the number of infected cows has been observed (Gonzalez et al., 1986; Farnsworth, 1993). This observation is not surprising as Staph. aureus in BTM also may originate from sites other than the mammary gland (environmental Staph. aureus), including the teat and udder skin, parlor, milking machine, air, and bedding (reviewed by Sears and McCarthy, 2003). According to Fox et al. (1991) and Zadoks et al. (2002), however, Staph. aureus associated with IMI differed from the environmental ones as shown by pulsed-field gel electrophoresis or phage typing and may therefore be considered mastitis irrelevant. To complicate matters further, some IMI-associated subtypes are not pathogenic (Fournier et al., 2008). The presented QPCR analytics, however, allow, for the first time, the highly sensitive detection of an actual IMIassociated and pathogenic subtype of Staph. aureus (GTB) in BTM, to the exclusion of all remaining and interfering subtypes.

Considering the analytical specificity of the assay, $100 \%$ of the GTB-positive strains $(\mathrm{n}=33$ ) were correctly detected. In addition, all remaining genotypes $(\mathrm{n}=38)$, CNS ( $\mathrm{n}=35$ ), other mastitis-causing and environmental bacteria $(\mathrm{n}=10)$, as well as further enterotoxin genes (sei, sej, seg), were safely excluded. Specificity remained the same when low and high concentrations of non-GTB targets were used. Furthermore, QPCR permitted detection of targets within a wide dynamic range, rendering dilution steps of samples unnecessary.

The high analytical specificity of the present GTBQPCR analytics was accompanied by a high analytical sensitivity as the detection limit for lukEB was $40 \mathrm{cfu}$ of Staph. aureus GTB per milliliter of milk. In addition, the repeatability of the lukEB-, sea-, and sed-QPCR assays were high. Based on the $\log _{10}$-transformed values, the intraassay variability of the 3 QPCR ranged from 0.9 to $2.9 \%$, the interassay variability ranged from 1.7 to $2.1 \%$. False-negative QPCR results caused by competitive or enzymatic inhibitors present in the sample NA were ruled out by inclusion and detection of an internal control (CDV-N gene). Multiplexing the 3 different QPCR was never considered. The main reasons for this decision were the reduced analytical sensitivity and efficiency of these QPCR assays, as well as the occurrence of false-negative results because of competing reactions (Henegariu et al., 1997).

To get an impression of how the novel methodology will perform in future extended field studies, simulation analyses (Figure 1) and an initial, exploratory field 
Table 5. Real bulk tank milk samples $(\mathrm{n}=33)$ : real-time quantitative PCR (QPCR) results for all detected targets, genotype (ribosomal spacer $\mathrm{PCR})$, and semiquantitative bacteriological findings after a 48-h incubation (10 $\mu \mathrm{L}$ inoculated)

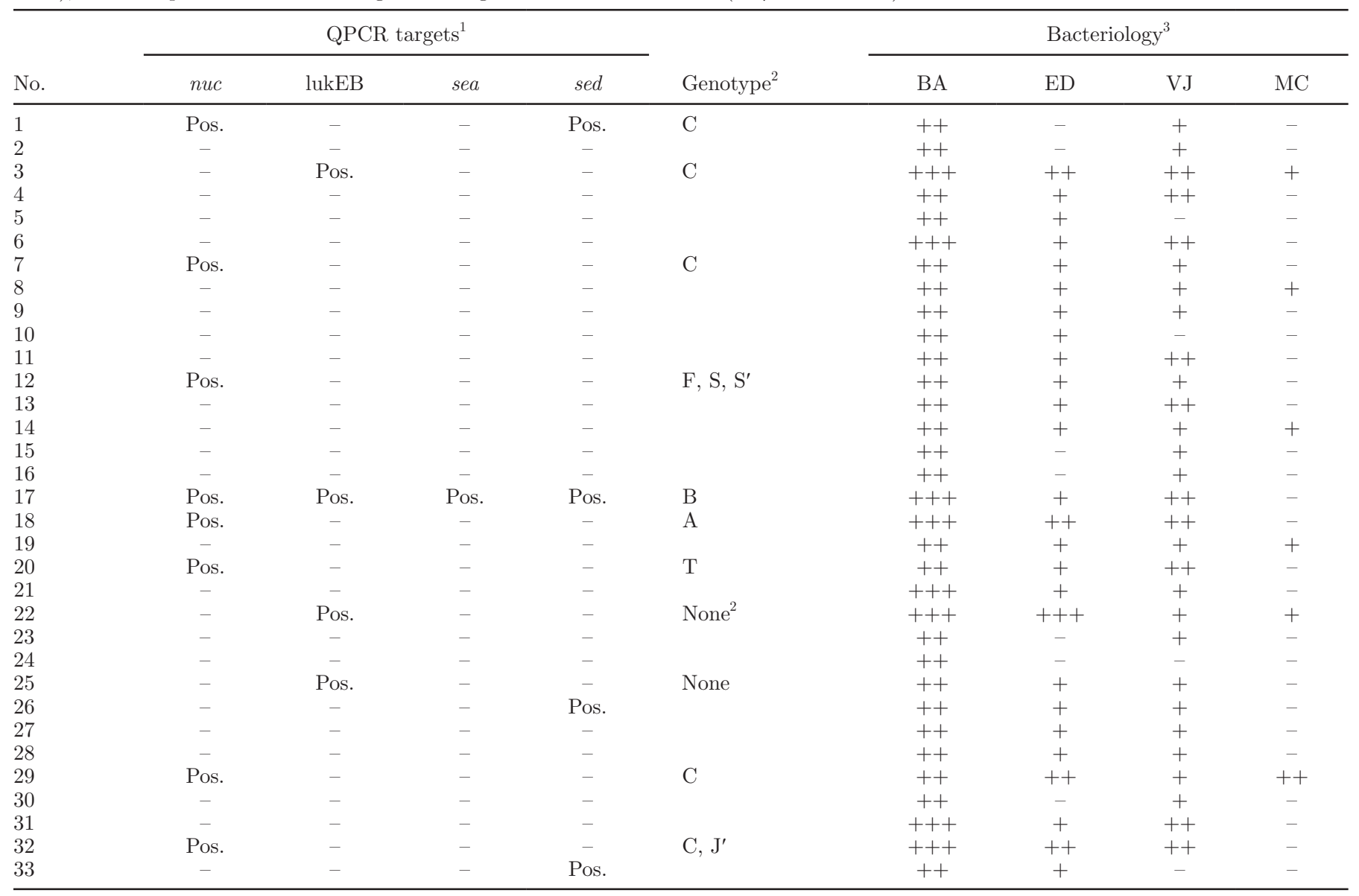

${ }^{1} n u c=$ thermonuclease gene; lukEB = point mutation within leucotoxin E gene, typical of GTB; sea = Staphylococcus aureus enterotoxin gene A; sed = Staph. aureus enterotoxin gene D; Pos. (positive) = amplification over limit of detection; - (negative) = no amplification.

${ }^{2}$ None $=$ no Staph. aureus colonies isolated.

${ }^{3} \mathrm{BA}=$ blood agar; $\mathrm{ED}=$ Edwards agar; VJ = Vogel-Johnson agar; MC = MacConkey agar; - , no growth (0 colony); +, weak growth (1-10 colonies); ++ , medium growth (10-100 colonies); +++ , intense growth (>100 colonies).

study were performed. An exponential dependency $\left(\mathrm{R}^{2}\right.$ $=0.861 ; P<0.001)$ between the logarithmic lukEB value and the simulated GTB cow prevalence was found. These investigations further revealed that the lowest p5BTM value accounted for 69 lukEB copies/reaction. As the detection limit of the lukEB-QPCR is 10 copies/ reaction, the minimal theoretical cow prevalence to be detected is $0.72 \%(5 \times 10 / 69)$, which translates to an expected detection rate of about 1 lukEB-positive cow among 138 cows using BTM. The p5BTM data also demonstrate the large range of lukEB values, resulting from single cow infections. If more cows are infected, pooling of milk averages the different amount of Staph. aureus, so that extreme values become impossible. Indeed, as shown in Figure 1, outliers were not observed when the cow prevalence was above $20 \%$.
Bacteriological analysis of the rBTM samples showed mid-range to intense growth on BA and limited growth of streptococci and related bacteria (ED agar). Only $18 \%$ of the samples were MC positive, which is an indicator of fecal contamination (coliforms). These results demonstrate that milking was properly carried out in most cases and that standards of hygiene were within an acceptable range. Considering VJ agar (NMC, 1999), staphylococci were observed in 29 of the $33 \mathrm{rBTM}$ samples (88\%). Compared with nuc QPCR (Table 5), which is highly sensitive and specific for Staph. aureus (Graber et al., 2007), VJ agar generated many more positive results, suggesting that at least some of them are false positive. These results are not surprising as a considerable number of CNS strains ferment mannitol and reduce tellurite (Holt et al., 1986). 
The subject of the present study was the development and technical evaluation of a novel methodology to detect Staph. aureus GTB in milk and to get an impression by simulation analyses and an initial field study of how this technology will perform in reality using BTM. Future extended field studies are necessary; in particular, investigations need to be performed focusing on the diagnostic and practical performance of the analytics as well as on the financial effect at the herd and country level when the novel methodology will be applied together with an appropriate control strategy.

\section{CONCLUSIONS}

The described QPCR analytics is a fast and potent method of screening easily available BTM samples for the Staph. aureus GTB. It is characterized by high analytical specificity and sensitivity as well as by high repeatability. Based on our simulation experiments and an initial field study, the methodology is expected to provide a powerful tool for the control of this contagious pathogen in the future.

\section{ACKNOWLEDGMENTS}

The study was supported by a grant of the Department of Clinical Veterinary Medicine, University of Berne, Switzerland, and the Swiss Federal Veterinary Office FVO. We thank M. Nydegger and E. Studer, veterinarians (Niederscherli, Switzerland), for their assistance in collecting BTM samples.

\section{REFERENCES}

Farnsworth, R. J. 1993. Microbiologic examination of bulk tank milk. Vet. Clin. North Am. Food Anim. Pract. 9:469-474.

Fournier, C., P. Kuhnert, J. Frey, R. Miserez, M. Kirchhofer, T. Kaufmann, A. Steiner, and H. U. Graber. 2008. Bovine Staphylococcus aureus: Association of virulence genes, genotypes and clinical outcome. Res. Vet. Sci. 85:439-448.

Fox, L. K., M. Gershman, D. D. Hancock, and C. T. Hutton. 1991. Fomites and reservoirs of Staphylococcus aureus causing intramammary infections as determined by phage typing: The effect of milking time hygiene practices. Cornell Vet. 81:183-193.

Gonzalez, R. N., D. E. Jasper, R. B. Bushnell, and T. B. Farver. 1986. Relationship between mastitis pathogen numbers in bulk tank milk and bovine udder infections in California dairy herds. J. Am. Vet. Med. Assoc. 189:442-445.

Graber, H. U., M. G. Casey, J. Naskova, A. Steiner, and W. Schaeren. 2007. Development of a highly sensitive and specific assay to detect Staphylococcus aureus in bovine mastitic milk. J. Dairy Sci. 90:4661-4669.

Graber, H. U., J. Naskova, E. Studer, T. Kaufmann, M. Kirchhofer, M. Brechbuhl, W. Schaeren, A. Steiner, and C. Fournier. 2009. Mastitis-related subtypes of bovine Staphylococcus aureus are characterized by different clinical properties. J. Dairy Sci. 92:1442-1451.

Henegariu, O., N. A. Heerema, S. R. Dlouhy, G. H. Vance, and P. H. Vogt. 1997. Multiplex PCR: Critical parameters and step-by-step protocol. Biotechniques 23:504-511.

Holt, J. G., P. H. A. Sneath, N. S. Maier, and M. E. Sharpe. 1986. Bergey's Manual of Systematic Bacteriology. Pages 1015-1035 in Genus IV. Staphylococcus. Vol. 2. Williams and Wilkins, Baltimore, MD

Jensen, M. A., J. A. Webster, and N. Straus. 1993. Rapid identification of bacteria on the basis of polymerase chain reaction-amplified ribosomal DNA spacer polymorphisms. Appl. Environ. Microbiol. 59:945-952.

Kirchhofer, M., L. Tavel, D. Strabel, C. Fournier, A. Steiner, H. U. Graber, and T. Kaufmann. 2007. Herd problem: Udder health. Retrospective study of farms assessed by the Swiss Bovine Health Service (BHS) from 1999 to 2004). Dtsch. Tierarztl. Wochenschr. 114:338-344.

Moret-Stalder, S., C. Fournier, R. Miserez, S. Albini, M. G. Doherr, M. Reist, W. Schaeren, M. Kirchhofer, H. U. Graber, A. Steiner, and T. Kaufmann. 2009. Prevalence study of Staphylococcus aureus in quarter milk samples of dairy cows in the Canton of Bern, Switzerland. Prev. Vet. Med. 88:72-76.

NMC. 1999. Laboratory Handbook on Bovine Mastitis. Rev. ed. National Mastitis Council, Madison, WI.

OIE World Organisation for Animal Health. 2008. Manual of Diagnostic Tests and Vaccines for Terrestrial Animals. 6th ed. OIE World Organisation for Animal Health, Paris, France.

Schällibaum, M. 1999. Mastitis pathogens isolated in Switzerland, 1987-1996. IDF Mastitis Newsl. 23:14.

Sears, P. M., and K. K. McCarthy. 2003. Management and treatment of staphylococcal mastitis. Vet. Clin. North Am. Food Anim. Pract. 19:171-185.

Sears, P. M., B. S. Smith, P. B. English, P. S. Herer, and R. N. Gonzalez. 1990. Shedding pattern of Staphylococcus aureus from bovine intramammary infections. J. Dairy Sci. 73:2785-2789.

Studer, E., W. Schaeren, J. Naskova, H. Pfaeffli, T. Kaufmann, M. Kirchhofer, A. Steiner, and H. U. Graber. 2008. A longitudinal field study to evaluate the diagnostic properties of a quantitative real-time polymerase chain reaction-based assay to detect Staphylococcus aureus in milk. J. Dairy Sci. 91:1893-1902.

Zadoks, R. N., W. B. van Leeuwen, D. Kreft, L. K. Fox, H. W. Barkema, Y. H. Schukken, and A. van Belkum. 2002. Comparison of Staphylococcus aureus isolates from bovine and human skin, milking equipment, and bovine milk by phage typing, pulsed-field gel electrophoresis, and binary typing. J. Clin. Microbiol. 40:38943902.

Zecconi, A., E. Binda, V. Borromeo, and R. Piccinini. 2005. Relationship between some Staphylococcus aureus pathogenic factors and growth rates and somatic cell counts. J. Dairy Res. 72:203-208. 